\title{
The boundary value problem of a
} three-dimensional generalized thermoelastic half-space subjected to moving rectangular heat source

\author{
Hamdy M. Youssef ${ }^{1,2^{*}}$ (D) and Eman A.N. Al-Lehaibi ${ }^{3}$
}

\author{
"Correspondence: \\ youssefanne2005@gmail.com \\ 'Mathematics Department, Faculty \\ of Education, Alexandria University, \\ Alexandria, Egypt \\ ${ }^{2}$ Mechanics Department, Faculty of \\ Engineering, Umm Al-Qura \\ University, Makkah, Saudi Arabia \\ Full list of author information is \\ available at the end of the article
}

\begin{abstract}
This paper deals with a new mathematical model of three-dimensional generalized thermoelasticity which has been improved using Lord-Shulman theory. The governing equations on non-dimensional forms have been applied to a three-dimensional half-space subjected to a rectangular moving heat source and traction-free surface by using the Laplace and double Fourier transform techniques. The inverses of the double Fourier and Laplace transforms have been calculated numerically by applying the complex formula of inversion of the transform by of the Fourier expansion method. The numerical results of the temperature increment, strain, stress, and displacement distributions have been represented in graphs for various values of the heat source speed parameter to show its effect on the thermo-mechanical waves. The heat source speed parameter leads to significant effects on both the thermal and mechanical waves.
\end{abstract}

Keywords: Three-dimensional modeling; Thermoelasticity; Laplace transforms; Double Fourier transforms; Moving heat source

\section{Nomenclature}

$\lambda, \mu$ Lame's parameters

$\rho$ Density

$C_{E}$ Specific heat of the material with constant strain

$t$ Time

$T$ Absolute temperature

$T_{0}$ Reference temperature

$\theta=\left(T-T_{0}\right)$ Temperature increment

$\alpha_{T}$ Linear thermal expansion coefficient

$\gamma=\alpha_{T}(3 \lambda+2 \mu)$

$\sigma_{i j}$ Stress tensor

$e_{i j}$ Strain tensor

$u_{i}$ Displacement components

$K$ Thermal conductivity

$\tau_{o}$ Relaxation time 


$$
\begin{aligned}
c_{o} & =\sqrt{\frac{\lambda+2 \mu}{\rho}} \\
\eta & =\frac{\rho C_{E}}{K} \\
\varepsilon & =\frac{\gamma^{2} T_{0}}{\rho C_{E}(\lambda+2 \mu)} \\
\beta & =\left(\frac{\lambda+2 \mu}{\mu}\right)^{1 / 2}
\end{aligned}
$$

\section{Introduction}

The difficulty in applications and in solving problems by using the mode of the Fourier transform is how the boundary conditions will be transformed. The important functions have been adapted by order and integrals of the eigenfunctions, depending on a definite system of coordinates (Morse, Feshbach [12]). To obtain the stresses and displacements, dissimilar derivatives of weight must be suited. Satisfying the limit conditions is a somewhat perplex work due to the appearance of elasticity coefficients in distinct and high powers in the denominators of the equations (Musii [13]). Podil'chuk and Kirichenko used the Fourier method (Podnil'Chuk, Kirichenko [16]) to construct a new approach for calculating the exact solutions to three-dimensional thermoelasticity problems in different coordinate systems.

A lot of numerical and computational methods can be found in viscoelasticity and thermoelasticity research (Danyluk et al. [2]; Oza et al. [14]; Vinogradov, Milton [21]). Laplace transform method is one of the well-known methods for thermoelasticity and viscoelasticity. De Chant used the numerical inversion rule and its limitations in the asymptotic and discontinuities methods (De Chant [3]). Temel got the solutions by applying the numerical method of Durbin using the Laplace transform inversions in the real space (Temel et al. [19]). Because of the intricacy of the determining relations, it is very difficult to gain the exact solutions of thermoelasticity, and the numerical approach has been preferred lately with the advances in the information processing system software incorporating the boundary and finite element methods (Mesquita, Coda [11]).

Youssef and Ezzat solved some models of three-dimensional thermoelasticity in the context of a different theory of thermoelasticity (Ezzat, Youssef [5-7]). Youssef studied many models of a thermoelastic material subjected to moving heat source in the context of different theorems of generalized thermoelasticity (Youssef [23, 24]). Youssef and Al-Lehaibi solved a problem of a three-dimensional generalized thermoelastic diffusion for a thermoelastic half-space subjected to rectangular thermal pulse (Youssef, Al-Lehaibi [25]). Parnell et al. employed the Wiener-Hopf technique and a modified Cagniard-de Hoop-type scheme, a rapidly convergent integral expression has been determined for a class of transient thermal mixed boundary value problems (Parnell et al. [15]). Kumar and Ailawalia studied the moving load response in a micropolar thermoelastic medium without energy dissipation possessing cubic symmetry (Kumar, Ailawalia [9]). Marin constructed an approach of a heat-flux dependent theory for a micropolar porous body, including voidage time derivative among the independent constitutive variables (Marin [10]). Sarkar and Lahiri discussed the interactions due to moving heat sources in a generalized thermoelastic half-space using LS model (Sarkar, Lahiri [17]). Tahouneh and Naei studied the effect of multi-directional nanocomposite materials on the vibrational response of thick shell panels with finite length and rested on two-parameter elastic foundations (Tahouneh, Naei [18]). The asymptotic behavior of solutions to a semilinear heat equation with homogeneous Neumann boundary conditions has been studied in (Ghisi 
et al. [8]). The global stability of rarefaction waves for the compressible Navier-Stokes equations has been investigated in (Duan et al. [4]).

In this work, a new mathematical model of three-dimensional generalized thermoelasticity will be constructed by using Lord-Shulman model. The governing equations on non-dimensional forms will be applied to a three-dimensional half-space subjected to a rectangular moving heat source and traction-free surface by using the Laplace and double Fourier transform techniques. The numerical results of the temperature increment, strain, stress, and displacement distributions will be represented in figures for various values of the heat source speed parameter to show its effect on the thermomechanical waves.

\section{The basic equations}

The system of partial differential equations of a homogeneous and isotropic thermoelastic medium based on the generalized thermoelasticity with one relaxation time and without any external body forces in undefined coordinates $\{i, j, k=1,2,3\}$ takes the following form (Ezzat, Youssef [5]; Youssef, Al-Lehaibi [25]):

The equations of motion are

$$
\mu u_{i, j j}+(\mu+\lambda) u_{j, i j}-(3 \lambda+2 \mu) \alpha \theta_{, i}=\rho \ddot{u}_{i}
$$

where $\theta=\left(T-T_{0}\right)$ is the increment of the temperature $T$ such that $\left|T-T_{0}\right| / T_{0} \ll 1$.

The heat equation is

$$
K \theta_{, i i}=\left(\frac{\partial}{\partial t}+\tau_{0} \frac{\partial^{2}}{\partial t^{2}}\right)\left[\rho C_{E} \theta+\gamma T_{0} e\right]-\left(1+\tau_{0} \frac{\partial}{\partial t}\right) Q .
$$

The constitutive relations are

$$
\sigma_{i j}=2 \mu e_{i j}+\lambda e_{k k} \delta_{i j}-\gamma \theta \delta_{i j} .
$$

The displacement relation with the strain takes the form

$$
e_{i j}=\frac{1}{2}\left(u_{i, j}+u_{j, i}\right) .
$$

\section{Problem formulation}

Assume an isotropic, homogeneous and thermoelastic body in three dimensions occupies the region $\Psi=\{x, y, z: 0 \leq x<\infty,-\infty<y<\infty,-\infty<z<\infty\}$ where the body is quiescent initially and is loaded thermally by a moving heat source with constant speed $v$, which starts from the bounding plane of the surface $x=0$ and moves along the $x$-axis when these surfaces are traction-free as in Fig. 1. The basic equations will be constructed in the context of Lord and Shulman model (L-S). The Cartesian coordinates $(x, y, z)$ will be used, and the displacement components of $u_{i}=(u, v, w)$ are set as follows (Ezzat, Youssef [5]; Youssef, Al-Lehaibi [25]):

The equations of motion are

$$
(\lambda+2 \mu) \frac{\partial^{2} u}{\partial x^{2}}+\mu\left(\frac{\partial^{2} u}{\partial y^{2}}+\frac{\partial^{2} u}{\partial z^{2}}\right)+(\lambda+\mu)\left(\frac{\partial^{2} v}{\partial x \partial y}+\frac{\partial^{2} w}{\partial x \partial z}\right)-\gamma \frac{\partial \theta}{\partial x}=\rho \ddot{u},
$$


Figure 1 A medium subjected to rectangular heat source moving with speed $v$

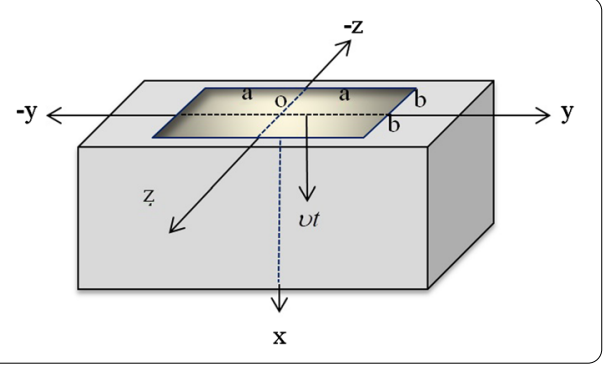

$$
\begin{aligned}
& (\lambda+2 \mu) \frac{\partial^{2} v}{\partial y^{2}}+\mu\left(\frac{\partial^{2} v}{\partial x^{2}}+\frac{\partial^{2} v}{\partial z^{2}}\right)+(\lambda+\mu)\left(\frac{\partial^{2} u}{\partial x \partial y}+\frac{\partial^{2} w}{\partial z \partial y}\right)-\gamma \frac{\partial \theta}{\partial y}=\rho \ddot{v}, \\
& (\lambda+2 \mu) \frac{\partial^{2} w}{\partial z^{2}}+\mu\left(\frac{\partial^{2} w}{\partial x^{2}}+\frac{\partial^{2} w}{\partial y^{2}}\right)+(\lambda+\mu)\left(\frac{\partial^{2} u}{\partial z \partial x}+\frac{\partial^{2} v}{\partial z \partial y}\right)-\gamma \frac{\partial \theta}{\partial z}=\rho \ddot{w} .
\end{aligned}
$$

The heat equation is

$$
\begin{aligned}
K\left(\frac{\partial^{2} \theta}{\partial x^{2}}+\frac{\partial^{2} \theta}{\partial y^{2}}+\frac{\partial^{2} \theta}{\partial z^{2}}\right)= & \rho C_{E}\left(\frac{\partial}{\partial t}+\tau_{0} \frac{\partial^{2}}{\partial t^{2}}\right) \theta \\
& +\gamma T_{o}\left(\frac{\partial}{\partial t}+\tau_{0} \frac{\partial^{2}}{\partial t^{2}}\right)\left(\frac{\partial u}{\partial x}+\frac{\partial v}{\partial y}+\frac{\partial w}{\partial z}\right)-\left(1+\tau_{0} \frac{\partial}{\partial t}\right) Q .
\end{aligned}
$$

The stress-strain (constitutive) relations are of the forms:

$$
\begin{aligned}
& \sigma_{x x}=2 \mu e_{x x}+\lambda e-\gamma \theta, \\
& \sigma_{y y}=2 \mu e_{y y}+\lambda e-\gamma \theta, \\
& \sigma_{z z}=2 \mu e_{z z}+\lambda e-\gamma \theta, \\
& \sigma_{x y}=2 \mu e_{x y}, \\
& \sigma_{x z}=2 \mu e_{x z},
\end{aligned}
$$

and

$$
\sigma_{y z}=2 \mu e_{y z}
$$

The strain components have the forms:

$$
\begin{aligned}
& e_{x x}=\frac{\partial u}{\partial x}, \quad e_{y y}=\frac{\partial v}{\partial y}, \quad e_{z z}=\frac{\partial w}{\partial z}, \\
& e_{x y}=\frac{1}{2}\left(\frac{\partial u}{\partial y}+\frac{\partial v}{\partial x}\right), \quad e_{x z}=\frac{1}{2}\left(\frac{\partial u}{\partial z}+\frac{\partial w}{\partial x}\right), \quad e_{y z}=\frac{1}{2}\left(\frac{\partial w}{\partial y}+\frac{\partial v}{\partial z}\right),
\end{aligned}
$$

and

$$
\frac{\partial u}{\partial x}+\frac{\partial v}{\partial y}+\frac{\partial w}{\partial z}=e_{x x}+e_{y y}+e_{z z}=e
$$

Equations (5)-(7) could be rewritten by using Eq. (17) as:

$$
\mu \nabla^{2} \frac{\partial u}{\partial x}+(\lambda+\mu) \frac{\partial^{2} e}{\partial x^{2}}-\gamma \frac{\partial^{2} \theta}{\partial x^{2}}=\rho \frac{\partial \ddot{u}}{\partial x},
$$




$$
\begin{aligned}
& \mu \nabla^{2} \frac{\partial v}{\partial y}+(\lambda+\mu) \frac{\partial^{2} e}{\partial y^{2}}-\gamma \frac{\partial^{2} \theta}{\partial y^{2}}=\rho \frac{\partial \ddot{v}}{\partial y} \\
& \mu \nabla^{2} \frac{\partial w}{\partial z}+(\lambda+\mu) \frac{\partial^{2} e}{\partial z^{2}}-\gamma \frac{\partial^{2} \theta}{\partial z^{2}}=\rho \frac{\partial \ddot{w}}{\partial z}
\end{aligned}
$$

where

$$
\nabla^{2}=\frac{\partial^{2}}{\partial x^{2}}+\frac{\partial^{2}}{\partial y^{2}}+\frac{\partial^{2}}{\partial z^{2}}
$$

The heat conduction equation takes the form

$$
K \nabla^{2} \theta=\rho C_{E}\left(\frac{\partial}{\partial t}+\tau_{0} \frac{\partial^{2}}{\partial t^{2}}\right) \theta+\gamma T_{o}\left(\frac{\partial}{\partial t}+\tau_{0} \frac{\partial^{2}}{\partial t^{2}}\right) e-\left(1+\tau_{0} \frac{\partial}{\partial t}\right) Q
$$

Now, the following dimensionless variables will be applied (Ezzat, Youssef [5-7]):

$$
\begin{aligned}
& \left(x^{*}, y^{*}, z^{*}\right)=c \eta(x, y, z), \quad\left(t^{*}, \tau_{0}^{*}\right)=c^{2} \eta\left(t, \tau_{o}\right), \quad \theta^{*}=\frac{\gamma \theta}{(\lambda+2 \mu)}, \\
& Q^{*}=\frac{Q}{K c^{2} \eta^{2} T_{o}}, \quad \sigma_{i j}^{*}=\frac{\sigma_{i j}}{\lambda+2 \mu}, \quad c=\sqrt{\frac{\lambda+2 \mu}{\rho}}, \quad \eta=\frac{\rho C_{E}}{K} .
\end{aligned}
$$

Thus, we obtain

$$
\begin{aligned}
& \beta \nabla^{2} \frac{\partial u}{\partial x}+(1-\beta) \frac{\partial^{2} e}{\partial x^{2}}-\frac{\partial^{2} \theta}{\partial x^{2}}=\frac{\partial \ddot{u}}{\partial x} \\
& \beta \nabla^{2} \frac{\partial v}{\partial y}+(1-\beta) \frac{\partial^{2} e}{\partial y^{2}}-\frac{\partial^{2} \theta}{\partial y^{2}}=\frac{\partial \ddot{v}}{\partial y}, \\
& \beta \nabla^{2} \frac{\partial w}{\partial z}+(1-\beta) \frac{\partial^{2} e}{\partial z^{2}}-\frac{\partial^{2} \theta}{\partial z^{2}}=\frac{\partial \ddot{w}}{\partial z}, \\
& \nabla^{2} \theta=\left(\frac{\partial}{\partial t}+\tau_{0} \frac{\partial^{2}}{\partial t^{2}}\right) \theta+\varepsilon_{1}\left(\frac{\partial}{\partial t}+\tau_{0} \frac{\partial^{2}}{\partial t^{2}}\right) e-\varepsilon_{2}\left(1+\tau_{0} \frac{\partial}{\partial t}\right) Q, \\
& \sigma_{x x}=2 \beta e_{x x}+(1-2 \beta) e-\theta, \\
& \sigma_{y y}=2 \beta e_{y y}+(1-2 \beta) e-\theta, \\
& \sigma_{z z}=2 \beta e_{z z}+(1-2 \beta) e-\theta, \\
& \sigma_{x y}=2 \beta e_{x y}, \\
& \sigma_{x z}=\beta e_{x z}, \\
& \sigma_{y z}=2 \beta e_{y z},
\end{aligned}
$$

where

$$
\beta=\frac{\mu}{\lambda+2 \mu}, \quad \varepsilon_{1}=\frac{\gamma^{2} T_{0}}{\rho C_{E}(\lambda+2 \mu)}, \quad \varepsilon_{2}=\frac{\gamma T_{0}}{(\lambda+2 \mu)} .
$$

Consider all the symbols without the stars to simplify the equations. 
By taking the sum of the Eqs. (22)-(24) and substituting Eq. (17), we get

$$
\nabla^{2} e-\nabla^{2} \theta=\ddot{e}
$$

We will assume the function $\sigma$ is the invariant stress, which is given as follows:

$$
\sigma=\frac{\sigma_{x x}+\sigma_{y y}+\sigma_{y y}}{3}
$$

By using Eqs. (26)-(28), we obtain

$$
\sigma=\alpha e-\theta
$$

where

$$
\alpha=\frac{3-4 \beta}{3}
$$

\section{Applying the Laplace transform on the governing equations}

We will be using Laplace transform, which is defined for any function $G(t)$ as follows:

$$
\bar{G}(s)=\int_{0}^{\infty} G(t) e^{-s t} d t
$$

Applying the transform to (35), we obtain:

$$
\begin{aligned}
& \beta \nabla^{2} \frac{\partial \bar{u}}{\partial x}+(1-\beta) \frac{\partial^{2} \bar{e}}{\partial x^{2}}-\frac{\partial^{2} \bar{\theta}}{\partial x^{2}}=s^{2} \frac{\partial \bar{u}}{\partial x}, \\
& \beta \nabla^{2} \frac{\partial \bar{v}}{\partial y}+(1-\beta) \frac{\partial^{2} \bar{e}}{\partial y^{2}}-\frac{\partial^{2} \bar{\theta}}{\partial y^{2}}=s^{2} \frac{\partial v}{\partial y}, \\
& \beta \nabla^{2} \frac{\partial \bar{w}}{\partial z}+(1-\beta) \frac{\partial^{2} \bar{e}}{\partial z^{2}}-\frac{\partial^{2} \bar{\theta}}{\partial z^{2}}=s^{2} \frac{\partial \bar{w}}{\partial z}, \\
& \nabla^{2} \bar{e}-\nabla^{2} \bar{\theta}=s^{2} \bar{e}, \\
& \nabla^{2} \bar{\theta}=\left(s+\tau_{0} s^{2}\right) \bar{\theta}+\varepsilon_{1}\left(s+\tau_{0} s^{2}\right) \bar{e}-\varepsilon_{2}\left(1+\tau_{0} s\right) \bar{Q}, \\
& \bar{\sigma}_{x x}=2 \beta \bar{e}_{x x}+(1-2 \beta) \bar{e}-\bar{\theta}, \\
& \bar{\sigma}_{y y}=2 \beta \bar{e}_{y y}+(1-2 \beta) \bar{e}-\bar{\theta}, \\
& \bar{\sigma}_{z z}=2 \beta \bar{e}_{z z}+(1-2 \beta) \bar{e}-\bar{\theta}, \\
& \bar{\sigma}_{x y}=2 \beta \bar{e}_{x y}, \\
& \bar{\sigma}_{x z}=\beta \bar{e}_{x z}, \\
& \bar{\sigma}_{y z}=2 \beta \bar{e}_{y z}, \\
& \bar{\sigma}=\alpha \bar{e}-\bar{\theta} .
\end{aligned}
$$


Using the Laplace transform, we applied the following initial conditions:

$$
\begin{aligned}
& \left.u(r, t)\right|_{t=0}=\left.v(r, t)\right|_{t=0}=\left.w(r, t)\right|_{t=0}=\left.\theta(r, t)\right|_{t=0}=0, \\
& \left.\frac{\partial u(r, t)}{\partial t}\right|_{t=0}=\left.\frac{\partial v(r, t)}{\partial t}\right|_{t=0}=\left.\frac{\partial w(r, t)}{\partial t}\right|_{t=0}=\left.\frac{\partial \theta(r, t)}{\partial t}\right|_{t=0}=0, \quad r=x, y, z .
\end{aligned}
$$

We assumed the medium is subjected to a rectangular heat source moving with constant speed and constant strength, releasing its energy continuously on a band of constant dimensions $2 a \times 2 b$ centered on the $y$-axis and $z$-axis, respectively, while moving with constant speed $v$ along the $x$-axis and being zero elsewhere as in Fig. 1.

Thus, the rectangular moving heat source is assumed to be of the following dimensionless form [22]:

$$
Q=Q_{o} \delta(x-v t) H(a-|y|) H(b-|z|),
$$

where $a$ and $b$ are constants, $Q_{o}$ is the heat source strength (constant), $\delta$ is the Dirac delta function, and $H(t)$ is the Heaviside function.

Applying the Laplace transform, we get

$$
Q=\frac{Q_{o}}{v} H(a-|y|) H(b-|z|) e^{-\left(\frac{s}{v}\right) x} .
$$

Eliminating $\bar{e}$ in Eqs. (39), (40) and (47), we get

$$
\nabla^{2} \bar{\sigma}=\alpha_{1} \bar{\theta}+\alpha_{2} \bar{\sigma}-\gamma_{1} H(a-|y|) H(b-|z|) e^{-\left(\frac{s}{v}\right) x}
$$

and

$$
\nabla^{2} \bar{\theta}=\alpha_{3} \bar{\theta}+\alpha_{4} \bar{\sigma}-\gamma_{2} H(a-|y|) H(b-|z|) e^{-\left(\frac{s}{v}\right) x},
$$

where

$$
\begin{aligned}
& \alpha_{1}=\frac{s^{2} \alpha-\left(s+\tau_{0} s^{2}\right)(1-\alpha)\left(\alpha+\varepsilon_{1}\right)}{\alpha}, \quad \alpha_{2}=\frac{s^{2} \alpha-\varepsilon_{1}(1-\alpha)}{\alpha}, \\
& \alpha_{3}=\frac{\left(s+\tau_{0} s^{2}\right)\left(\alpha+\varepsilon_{1}\right)}{\alpha}, \quad \alpha_{4}=\frac{\varepsilon_{1}\left(s+\tau_{0} s^{2}\right)}{\alpha}, \\
& \gamma_{1}=\varepsilon_{2}\left(1+\tau_{0} s\right)(\alpha-1) \frac{Q_{o}}{v}, \quad \gamma_{2}=\varepsilon_{2}\left(1+\tau_{0} s\right) \frac{Q_{o}}{v} .
\end{aligned}
$$

\section{Applying the double Fourier transform}

The double Fourier transform for any function $f(x, y, z)$ is defined as follows:

$$
F[\bar{f}(x, y, z, s)]=\tilde{\bar{f}}(x, p, q, s)=\frac{1}{2 \pi} \int_{-\infty}^{\infty} \int_{-\infty}^{\infty} \bar{f}(x, y, z, s) e^{-i(p y+q z)} d y d z,
$$

where the inverse of the double Fourier transform takes the form

$$
F^{-1}[\tilde{\tilde{f}}(x, p, q, s)]=\bar{f}(x, y, z, s)=\frac{1}{2 \pi} \int_{-\infty}^{\infty} \int_{-\infty}^{\infty} \tilde{\tilde{f}}(x, p, q, s) e^{i(p y+q z)} d p d q .
$$


Thus, we have

$$
F\left[\nabla^{2} \bar{f}(x, y, z, s)\right]=\left(\frac{d^{2}}{d x^{2}}-q^{2}-p^{2}\right) \tilde{\tilde{f}}(x, p, q, s) .
$$

Applying the transforms to (53) and (55), we get the following ordinary differential equations:

$$
\frac{d^{2} \tilde{\bar{\sigma}}}{d x^{2}}=\alpha_{1} \tilde{\bar{\theta}}+\beta_{1} \tilde{\bar{\sigma}}-\gamma_{3} e^{-\left(\frac{s}{v}\right) x}
$$

and

$$
\frac{d^{2} \tilde{\bar{\theta}}}{d x^{2}}=\beta_{2} \tilde{\bar{\theta}}+\alpha_{4} \tilde{\bar{\sigma}}-\gamma_{4} e^{-\left(\frac{s}{v}\right) x},
$$

where

$$
\begin{aligned}
& \beta_{1}=p^{2}+q^{2}+\alpha_{2}, \quad \beta_{2}=p^{2}+q^{2}+\alpha_{3}, \\
& \gamma_{3}=\frac{2}{\pi} \frac{\gamma_{1} \sin (q a) \sin (p a)}{q p}, \text { and } \quad \gamma_{4}=\frac{2}{\pi} \frac{\gamma_{2} \sin (q b) \sin (p b)}{q p} .
\end{aligned}
$$

Eliminating $\tilde{\bar{\sigma}}$ from Eqs. (56) and (57), we get

$$
\left[\frac{d^{4}}{d x^{4}}-\left(\beta_{1}+\beta_{2}\right) \frac{d^{2}}{d x^{2}}+\left(\beta_{1} \beta_{2}-\alpha_{1} \alpha_{4}\right)\right] \tilde{\bar{\theta}}=-\beta_{5} e^{-\frac{s}{v} x} .
$$

Similarly, eliminating $\tilde{\bar{\theta}}$ from Eqs. (56) and (57), we obtain

$$
\left[\frac{d^{4}}{d x^{4}}-\left(\beta_{1}+\beta_{2}\right) \frac{d^{2}}{d x^{2}}+\left(\beta_{1} \beta_{2}-\alpha_{1} \alpha_{4}\right)\right] \tilde{\bar{\sigma}}=-\beta_{6} e^{-\frac{s}{v} x},
$$

where

$$
\beta_{5}=\gamma_{4}\left(\frac{s^{2}}{v^{2}}-\beta_{1}\right)+\alpha_{4} \gamma_{3}, \quad \beta_{6}=\gamma_{3}\left(\frac{s^{2}}{v^{2}}-\beta_{2}\right)+\gamma_{4} \alpha_{1} .
$$

The solution of Eq. (58) takes the form

$$
\tilde{\bar{\theta}}=A_{1} e^{-k_{1} x}+A_{2} e^{-k_{2} x}-A_{3} e^{-\frac{s}{v} x},
$$

where

$$
A_{3}=\frac{\beta_{5}}{\left(\frac{s^{2}}{v^{2}}-k_{1}^{2}\right)\left(\frac{s^{2}}{v^{2}}-k_{2}^{2}\right)} .
$$

The solution of Eq. (59) takes the form

$$
\tilde{\tilde{\sigma}}=B_{1} e^{-k_{1} x}+B_{2} e^{-k_{2} x}-B_{3} e^{-\frac{s}{v} x},
$$


where

$$
B_{3}=\frac{\beta_{6}}{\left(\frac{s^{2}}{v^{2}}-k_{1}^{2}\right)\left(\frac{s^{2}}{v^{2}}-k_{2}^{2}\right)},
$$

with $A_{1}, A_{2}, B_{1}$, and $B_{2}$ being some parameters, and $\pm k_{1}^{2}$ and $\pm k_{2}^{2}$ the roots of the characteristic equation:

$$
k^{4}-L k^{2}+M=0
$$

where $L=\beta_{1}+\beta_{2}$ and $M=\beta_{1} \beta_{2}-\alpha_{1} \alpha_{4}$, which satisfy the relations:

$$
k_{1}^{2}+k_{2}^{2}=\beta_{1}+\beta_{2}, \quad k_{1}^{2} k_{2}^{2}=\beta_{1} \beta_{2}-\alpha_{1} \alpha_{4} .
$$

From Eqs. (60), (61) and (56), we get

$$
B_{1}=\frac{\left(k_{1}^{2}-\alpha_{1}\right)}{\beta_{1}} A_{1}, \quad B_{2}=\frac{\left(k_{2}^{2}-\alpha_{1}\right)}{\beta_{1}} A_{2} .
$$

Hence, we have

$$
\tilde{\bar{\sigma}}=\frac{\left(k_{1}^{2}-\alpha_{1}\right) A_{1}}{\beta_{1}} e^{-k_{1} x}+\frac{\left(k_{2}^{2}-\alpha_{1}\right) A_{2}}{\beta_{1}} e^{-k_{2} x}-B_{3} e^{-\frac{s}{v} x} .
$$

To finalize the solution, we have to obtain the parameters $A_{1}, A_{2}$ by applying certain boundary conditions. We consider that the bounding plane of the surface is traction-free and it has no external thermal loading, which gives, by using all the above transformations, the following conditions:

$$
\tilde{\tilde{\sigma}}(0, q, p, s)=\tilde{\bar{\theta}}(0, y, z, t)=0 .
$$

Substituting (66) into Eqs. (60) and (61), we get the following system:

$$
\begin{aligned}
& A_{1}+A_{2}=A_{3}, \\
& \left(k_{1}^{2}-\alpha_{1}\right) A_{1}+\left(k_{2}^{2}-\alpha_{1}\right) A_{2}=\beta_{1} B_{3} .
\end{aligned}
$$

The solution of the above system gives

$$
A_{1}=\frac{A_{3}\left(k_{2}^{2}-\alpha_{1}\right)-\beta_{1} B_{3}}{k_{2}^{2}-k_{1}^{2}}, \quad A_{2}=-\frac{A_{3}\left(k_{1}^{2}-\alpha_{1}\right)-\beta_{1} B_{3}}{k_{2}^{2}-k_{1}^{2}} .
$$

Hence, we have the solutions in Fourier and Laplace transform domain as follows:

$$
\begin{aligned}
\tilde{\bar{\theta}}(x, p, q, s)= & \frac{A_{3}\left(k_{2}^{2}-\alpha_{1}\right)-\beta_{1} B_{3}}{\left(k_{2}^{2}-k_{1}^{2}\right)} e^{-k_{1} x}-\frac{A_{3}\left(k_{1}^{2}-\alpha_{1}\right)-\beta_{1} B_{3}}{\left(k_{2}^{2}-k_{1}^{2}\right)} e^{-k_{2} x}-A_{3} e^{-\frac{s}{v} x}, \\
\tilde{\bar{\sigma}}(x, p, q, s)= & \frac{\left(k_{1}^{2}-\alpha_{1}\right)\left[A_{3}\left(k_{2}^{2}-\alpha_{1}\right)-\beta_{1} B_{3}\right]}{\beta_{1}\left(k_{2}^{2}-k_{1}^{2}\right)} e^{-k_{1} x} \\
& -\frac{\left(k_{2}^{2}-\alpha_{1}\right)\left[A_{3}\left(k_{1}^{2}-\alpha_{1}\right)-\beta_{1} B_{3}\right]}{\beta_{1}\left(k_{2}^{2}-k_{1}^{2}\right)} e^{-k_{2} x}-B_{3} e^{-\frac{s}{v} x},
\end{aligned}
$$


and

$$
\tilde{\bar{e}}(x, p, q, s)=\frac{1}{\alpha}[\tilde{\bar{\sigma}}(x, p, q, s)+\tilde{\bar{\theta}}(x, p, q, s)] .
$$

\section{Inversion of both Fourier and Laplace transforms}

To get the final solution in its original variables, we should calculate the inverse of the double Fourier and Laplace transforms in Eqs. (70)-(72). These expressions may be formally written as functions of $x$, and all the parameters of the Fourier and Laplace transforms, namely $p, q$, and $s$, in the form $\tilde{\bar{f}}(x, q, p, s)$ (Ezzat, Youssef [5-7]).

In the beginning, we invert the double Fourier transforms using the formula in (54) which gives the expression $\bar{f}(x, y, z, s)$ in the Laplace transform domain as follows (Ezzat, Youssef [5]):

$$
\begin{aligned}
\bar{f}(x, y, z, s) & =F^{-1}[\tilde{\bar{f}}(x, p, q, s)] \\
& =\frac{1}{2 \pi} \int_{-\infty}^{\infty} \int_{-\infty}^{\infty} \tilde{\bar{f}}(x, p, q, s) e^{i(p y+q z)} d p d q \\
& =\frac{2}{\pi} \int_{0}^{\infty} \int_{0}^{\infty}\left[\cos (p y+q z) \tilde{\bar{f}}_{e}+i \sin (p y+q z) \tilde{\bar{f}}_{o}\right] d p d q
\end{aligned}
$$

where $\tilde{\bar{f}}_{o}$ and $\tilde{\bar{f}}_{e}$ denote the odd and even parts of the function $\tilde{\bar{f}}(x, q, p, s)$, respectively. To invert the Laplace transform, the technique of the Riemann-sum approximation will be applied as (Tzou [20]):

$$
g(t)=\frac{e^{\kappa t}}{t}\left[\frac{1}{2} \bar{g}(\kappa)+\operatorname{Re} \sum_{n=1}^{N}(-1)^{n} \bar{g}\left(\kappa+\frac{i n \pi}{t}\right)\right]
$$

Here Re means the real part and $i=\sqrt{-1}$. Almost all the computational experiments have shown that the value of $\kappa$ comes from the relation $\kappa t \approx 4$.7, which gives faster convergence, (Tzou [20]).

\section{Numerical results and discussion}

Copper was taken for the numerical calculations, and the constants of the material have been taken as follows (Ezzat, Youssef [5-7]; Abbas, Youssef [1]):

$$
\begin{aligned}
& K=386 \mathrm{~N} / \mathrm{K} \mathrm{sec}, \quad \alpha_{T}=1.78 \times 10^{-5} \mathrm{~K}^{-1}, \quad C_{E}=383.1 \mathrm{~m}^{2} / \mathrm{K}, \\
& \eta=8886.73 \mathrm{~m} / \mathrm{sec}^{2}, \quad T_{0}=293 \mathrm{~K}, \quad \mu=3.86 \times 10^{10} \mathrm{~N} / \mathrm{m}^{2}, \\
& \lambda=7.76 \times 10^{10} \mathrm{~N} / \mathrm{m}^{2}, \quad \rho=8954 \mathrm{~kg} / \mathrm{m}^{3}, \quad \tau_{0}=0.33 \times 10^{-15} \mathrm{sec} .
\end{aligned}
$$

Thus, we get the following non-dimensional parameters:

$$
\tau_{0}=0.002, \quad \beta=0.25, \quad \alpha=0.67, \quad \varepsilon_{1}=0.0168, \quad \varepsilon_{2}=0.010444 .
$$

The computations were running out for non-dimensional time $t=0.1$, length $a=b=$ 1.0 , and heat source intensity $Q_{0}=1.0$. The temperature, strain, stress, and displacement distributions are shown in graphs. 


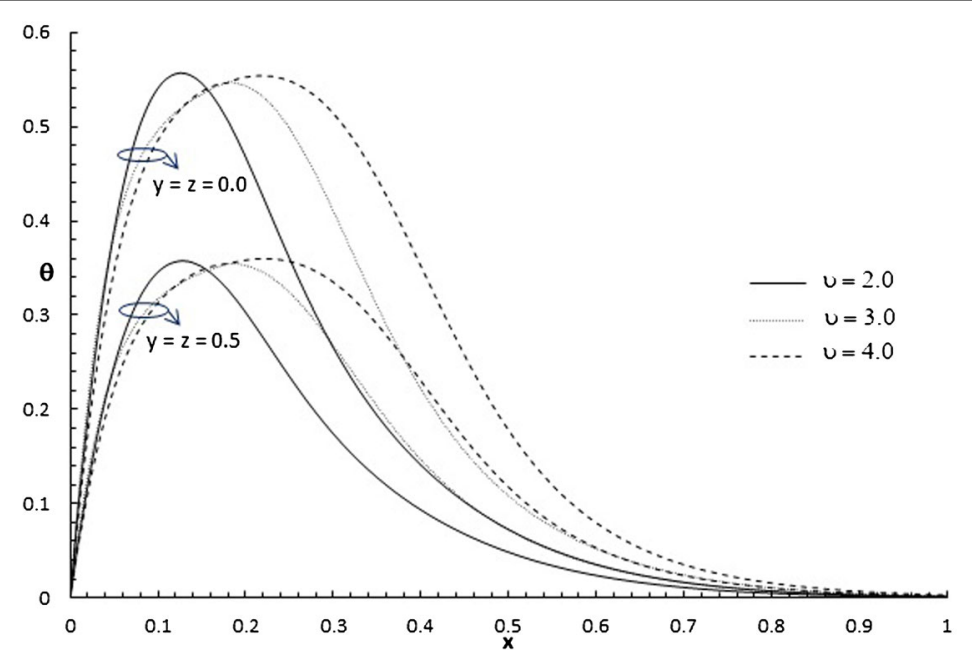

Figure 2 The temperature increment distribution for various values of heat source speed



Figure 3 The stress distribution for various values of heat source speed

Figures $2-5$ represent the temperature increment, stress, strain, and displacement component $u_{x}$ distributions, respectively, for various values of heat source speed $v=$ $(2.0,3.0,4.0)$ and a wide range of distance $x(0 \leq x \leq 1)$ when $y=z=0.0$ and $y=z=0.5$ to show the effect of the heat source speed on all the studied functions.

The value of speed of the heat source has significant effects on all the studied functions. Increasing the values of the speed of the heat source leads to decreasing the temperature increment values and the absolute values of the stress before the peaks, while after the peaks, the increase of the speed of the heat source leads to increases in them. Increasing the values of the speed of the heat source leads to decreasing of the strain and the displacement values. The results and the figures have max or min points because the effect of the heat source is working in the interval $x<v t$ so the temperature increment increases rapidly, while in the interval $x \geq v t$ the heat source disappears, which makes the temperature increment decrease rapidly. Accordingly, all the other functions will be affected by this attitude. In other words, the value of the position $x=v t$ is a critical position because 



it separates between the existence of the heat source and its disappearance. The effects of the moving heat source of the current results agree with the results in (Youssef [22-24]; Youssef, Al-Lehaibi [25]).

Figures 6-9 represent the temperature increment, stress, strain, and displacement component $u_{x}$ distributions, respectively, for a wide range of heat source speed $v$ and a wide range of distance $x$ when $y=z=0.5$ to illustrate the effect of the heat source speed on all the studied functions. These figures agree with the results of Figs. 2-5.

\section{Conclusions}

- The value of the speed of the heat source has significant effects on the temperature increment, stress, strain, and displacement distributions.

- The values of the peak points for all the studied functions increase when the speed of the moving heat source increases. 


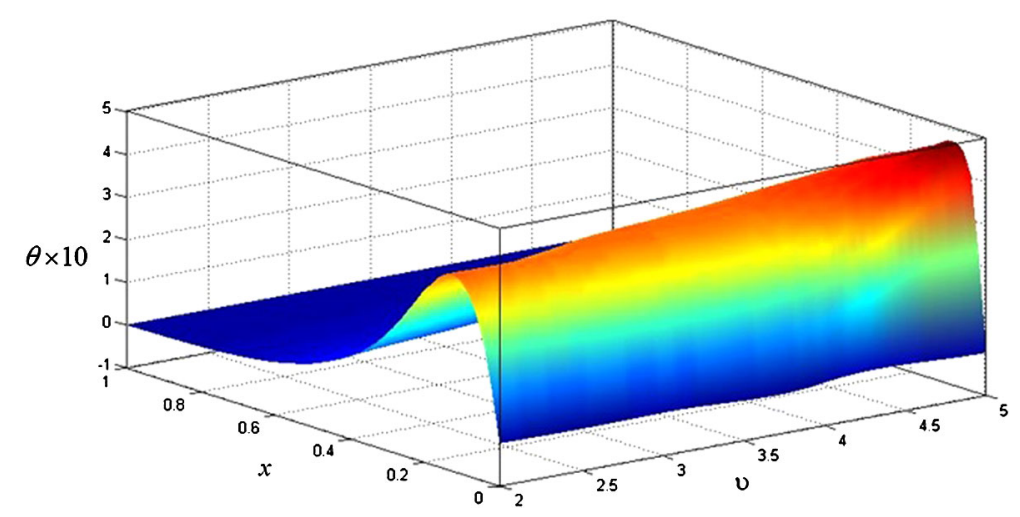

Figure 6 The temperature increment distribution for a wide range of heat source speed when $y=z=0.5$

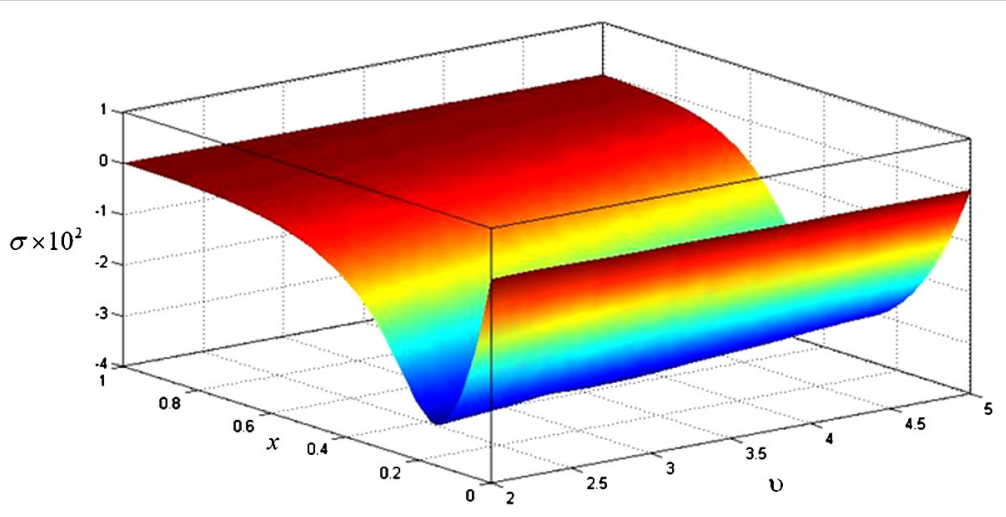

Figure 7 The stress distribution for a wide range of heat source speed when $y=z=0.5$

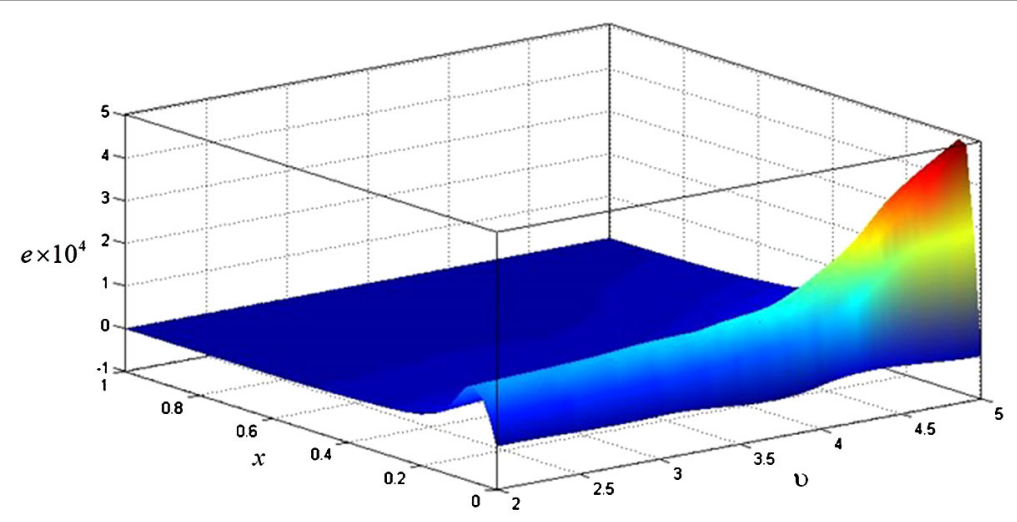

Figure 8 The strain distribution for a wide range of heat source speed when $y=z=0.5$

- The temperature increment, stress, strain, and displacement have different behavior before and after the critical position $x=v t$, which separates between the existence of the heat source and its disappearance.

- The positions along the $y$ and $z$ axes have significant effects on all the studied functions. 




Figure 9 The displacement component $u_{x}$ distribution for a wide range of heat source speed when $y=z=0.5$

\section{Acknowledgements}

Not applicable.

Funding

This work has not been supported by any fund from anywhere.

\section{Availability of data and materials}

Data sharing is not applicable to this article as no datasets were generated or analyzed during the current study.

\section{Competing interests}

The authors declare that they have no competing interests.

\section{Authors' contributions}

HY constructed the problem and the governing equations and advised the co-author on getting the numerical solutions. $\mathrm{HY}$ wrote the following sections: abstract, introduction, the basic equation, the formulation of the problem, conclusions, and reviewed all the work. EA solved the problem, applied the Laplace transform on the governing equations and its inversions, prepared figures of the numerical results, and wrote the remaining sections. All authors read and approved the final manuscript.

\section{Author details}

${ }^{1}$ Mathematics Department, Faculty of Education, Alexandria University, Alexandria, Egypt. ${ }^{2}$ Mechanics Department, Faculty of Engineering, Umm Al-Qura University, Makkah, Saudi Arabia. ${ }^{3}$ Mathematics Department, Al-Lith University College, Umm Al-Qura University, Al Lith, Saudi Arabia.

\section{Publisher's Note}

Springer Nature remains neutral with regard to jurisdictional claims in published maps and institutional affiliations.

Received: 4 September 2018 Accepted: 2 January 2019 Published online: 15 January 2019

\section{References}

1. Abbas, I.A., Youssef, H.M.: Two-dimensional fractional order generalized thermoelastic porous material. Lat. Am. J. Solids Struct. 12(7), 1415-1431 (2015)

2. Danyluk, M., Geubelle, P., Hilton, H.: Two-dimensional dynamic and three-dimensional fracture in viscoelastic materials. Int. J. Solids Struct. 35(28-29), 3831-3853 (1998)

3. De Chant, L.: Impulsive displacement of a quasi-linear viscoelastic material through accurate numerical inversion of the Laplace transform. Comput. Math. Appl. 43(8-9), 1161-1170 (2002)

4. Duan, R., Liu, H., Zhao, H.: Nonlinear stability of rarefaction waves for the compressible Navier-Stokes equations with large initial perturbation. Trans. Am. Math. Soc. 361(1), 453-493 (2009)

5. Ezzat, M.A., Youssef, H.M.: Three-dimensional thermal shock problem of generalized thermoelastic half-space. Appl. Math. Model. 34(11), 3608-3622 (2010)

6. Ezzat, M.A., Youssef, H.M.: Two-temperature theory in three-dimensional problem for thermoelastic half space subjected to ramp type heating. Mech. Adv. Mat. Struct. 21(4), 293-304 (2014)

7. Ezzat, M.A., Youssef, H.M.: Three-dimensional thermo-viscoelastic material. Mech. Adv. Mat. Struct. 23(1), 108-116 (2016)

8. Ghisi, M., Gobbino, M., Haraux, A.: A concrete realization of the slow-fast alternative for a semilinear heat equation with homogeneous Neumann boundary conditions. Adv. Nonlinear Anal. 7(3), 375-384 (2018)

9. Kumar, R., Ailawalia, P.: Moving load response in micropolar thermoelastic medium without energy dissipation possessing cubic symmetry. Int. J. Solids Struct. 44(11-12), 4068-4078 (2007) 
10. Marin, M.: An approach of a heat-flux dependent theory for micropolar porous media. Meccanica 51(5), 1127-1133 (2016)

11. Mesquita, A., Coda, H.: A boundary element methodology for viscoelastic analysis: part I with cells. Appl. Math. Model. 31(6), 1149-1170 (2007)

12. Morse, P.M., Feshbach, H.: Methods of Theoretical Physics, vol. II (2010)

13. Musii, R.: Equations in stresses for two-and three-dimensional dynamic problems of thermoelasticity in spherical coordinates. Mater. Sci. 39(1), 48-53 (2003)

14. Oza, A., Vanderby, R., Lakes, R.S.: Generalized solution for predicting relaxation from creep in soft tissue: application to ligament. Int. J. Mech. Sci. 48(6), 662-673 (2006)

15. Parnell, W.J., Nguyen, V.-H., Assier, R., Naili, S., Abrahams, I.D.: Transient thermal mixed boundary value problems in the half-space. SIAM J. Appl. Math. 76(3), 845-866 (2016)

16. Podnil'Chuk, I.N., Kirichenko, A.: Thermoelastic deformation of a parabolic cylinder. Vychisl. Prikl. Mat. 67, 80-88 (1989)

17. Sarkar, N., Lahiri, A.: Interactions due to moving heat sources in generalized thermoelastic half-space using LS model. Int. J. Appl. Mech. Eng. 18(3), 815-831 (2013)

18. Tahouneh, $\mathrm{V}$., Naei, M.H.: The effect of multi-directional nanocomposite materials on the vibrational response of thick shell panels with finite length and rested on two-parameter elastic foundations. Int. J. Adv. Struct. Eng. 8(1), 11-28 (2016)

19. Temel, B., Çalim, F.F., Tütüncü, N.: Quasi-static and dynamic response of viscoelastic helical rods. J. Sound Vib. 271(3), 921-935 (2004)

20. Tzou, D.: Macro to Micro Heat Transfer. Taylor \& Francis, Washington (1996)

21. Vinogradov, V., Milton, G.: The total creep of viscoelastic composites under hydrostatic or antiplane loading. J. Mech. Phys. Solids 53(6), 1248-1279 (2005)

22. Youssef, H.: A two-temperature generalized thermoelastic medium subjected to a moving heat source and ramp-type heating: a state-space approach. J. Mech. Mater. Struct. 4(9), 1637-1649 (2010)

23. Youssef, H.M.: Generalized thermoelastic infinite medium with cylindrical cavity subjected to moving heat source. Mech. Res. Commun. 36(4), 487-496 (2009)

24. Youssef, H.M.: Two-temperature generalized thermoelastic infinite medium with cylindrical cavity subjected to moving heat source. Arch. Appl. Mech. 80(11), 1213-1224 (2010)

25. Youssef, H.M., Al-Lehaibi, E.A.: Three-dimensional generalized thermoelastic diffusion and application for a thermoelastic half-space subjected to rectangular thermal pulse. J. Therm. Stresses 41(8), 1008-1021 (2018)

\section{Submit your manuscript to a SpringerOpen ${ }^{\circ}$ journal and benefit from:}

- Convenient online submission

- Rigorous peer review

Open access: articles freely available online

- High visibility within the field

- Retaining the copyright to your article

Submit your next manuscript at $>$ springeropen.com 